\title{
Dolor torácico y alteración electrocardiográfica
}

\author{
Alejandro Paredes, Alex Bittner, Ismael Vergara. \\ Unidad de Electrofisiología y Arritmología Cardíaca, División de Enfermedades Cardiovasculares \\ Pontificia Universidad Católica de Chile.
}

\section{A man with anginal pain and an abnormal EKG}

A 57 year-old man, smoker, with high blood pressure, presented to the emergency unit with intermittent and brief typical anginal pain in the preceding 2 days. Baseline physical examination was nor- mal. Figure 1 depicts de EKG recorded upon admission. Biomarkers for acute coronary syndrome were negative.

Keywords: Anginal pain, EKG.

Correspondencia:

Dr. Alejandro Paredes

División de Enfermedades Cardiovasculares PUC.

Diagonal Paraguay $3627^{\circ}$ piso

Fono: (+56 02) 23543644

faparedes@puc.cl 


\section{Caso clínico:}

Se presenta el caso clínico de un paciente de sexo masculino, de 57 años con antecedentes de tabaquismo e hipertensión arterial con control y tratamiento irregular. Consulta en el servicio de urgencias de nuestra institución por cuadro de dolor torácico anginoso, intermitente, de segundos a minutos de duración y de 2 días de evolución. Por la persistencia de los síntomas decide consultar. Al ingreso, el examen físico no presentó hallazgos de significado patológico. El ECG registrado en la sala de Urgencias, estando el paciente sin dolor se presenta en la Figura 1.

Los resultados de laboratorio, incluyendo Troponina $\mathrm{T}$ ultrasensible y $\mathrm{CK}$ total/MB, resultaron negativos.

En vista de los factores de riesgo cardiovascular presentados por el paciente, las características clínicas del dolor torácico y los hallazgos electrocardiográficos encontrados, se decide hospitalizar al paciente. Al día siguiente se realizó un estudio invasivo que permitió establecer el diagnóstico definitivo.

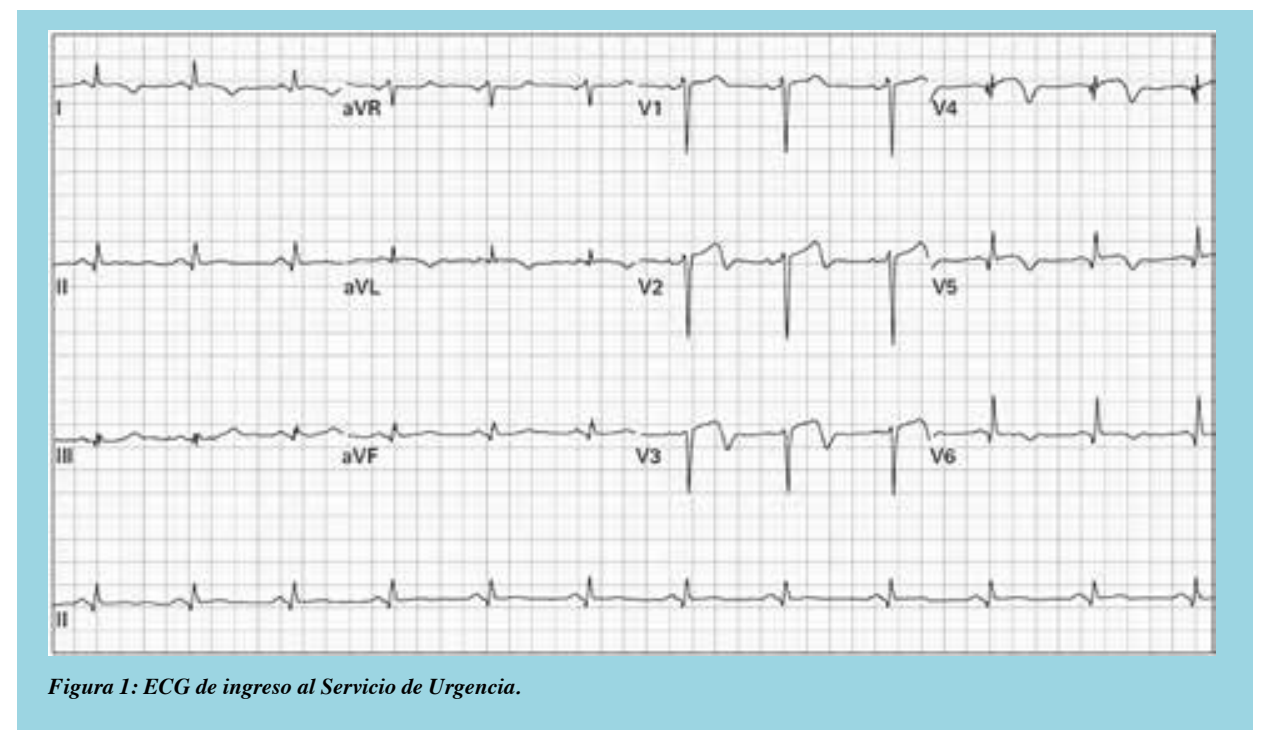


El estudio coronariográfico se realizó al día siguiente al ingreso y mostró una estenosis crítica de la arteria descendente anterior en su segmento proximal (Figura 2) la cual fue exitosamente tratada con el implante de un stent liberador de drogas. El paciente evolucionó satisfactoriamente siendo dado de alta al cuarto día.

\section{Discusión:}

El síndrome de Wellens (SW) también llamado "left anterior descending artery T-wave síndrome", fue descrito originalmente por Wellens et al., en el año $1982^{1}$. Consiste en la presencia de un patrón electrocardiográfico con cambios en la onda $\mathrm{T}$ en las derivaciones precordiales (inversión profunda simétrica de la onda $\mathrm{T}$ u ondas $\mathrm{T}$ bifásicas generalmente en V2-3, con progresión de la onda $\mathrm{R}$ conservada, ausencia de ondas $\mathrm{Q}$ patológicas y de elevación del ST), asociados a síntomas previos de angina inestable. Se manifiesta cuando el paciente está libre de dolor, y sin elevación (o mínima) de las enzimas cardíacas (Tabla 1). Estos hallazgos se asocian a enfermedad significativa del segmento proximal de la arteria descendente anterior (ADA) y pueden predecir el desarrollo posterior de un infarto agudo al miocardio como parte de su evolución, con alta sensibilidad y especificidad ${ }^{2}$.

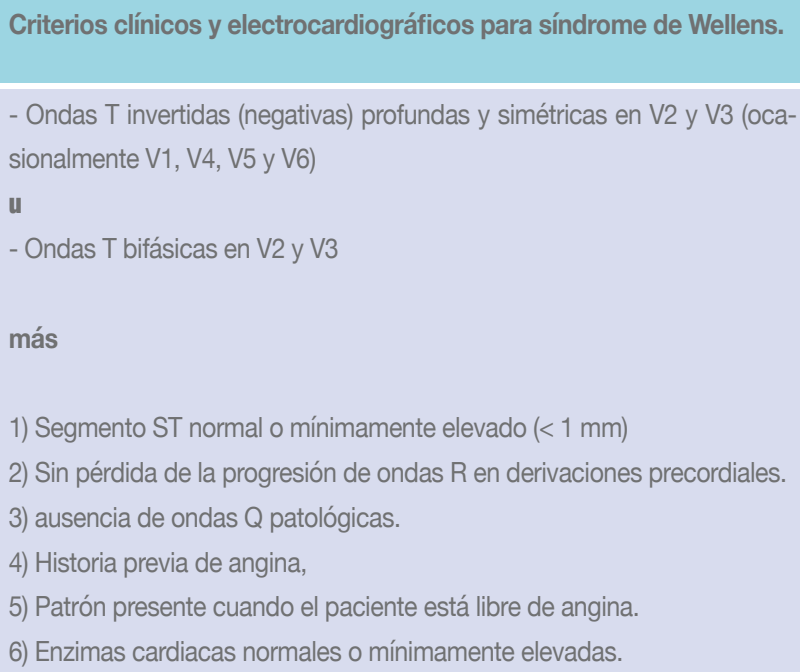

Tabla 1: Criterios diagnósticos para síndrome de Wellens.

La realización de pruebas de isquemia en este grupo de pacientes puede ser riesgoso; por lo que se sugiere una estrategia invasiva precoz.

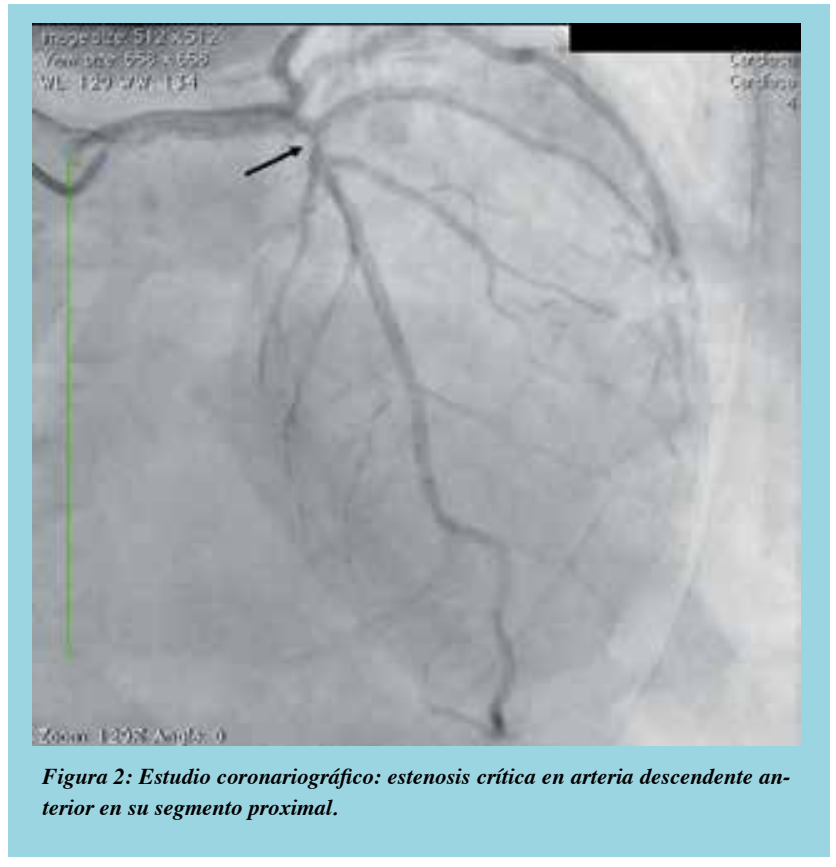

\section{Clásicamente se reconocen dos variantes del SW:}

- El tipo 1 corresponde a la minoría de los casos (24\%) y se caracteriza por el hallazgo de onda T bifásica (+/-) en las derivaciones $\mathrm{V} 2 \mathrm{y} \mathrm{V} 3$, pudiendo extenderse al resto de la pared anterior.

- El tipo 2 representa la mayoría de los casos (76\%) y muestra onda $\mathrm{T}$ con inversión profunda y simétrica, típicamente, en las derivaciones $\mathrm{V} 2$ y V3, pudiendo producirse en V1-V4 y, eventualmente, en V5 y V6.

En un grupo de 118 pacientes hospitalizados por angina inestable, en quienes se realizó seguimiento electrocardiográfico y estudio coronariográfico durante la hospitalización, Haines et al., describieron una sensibilidad, especificidad y valor predictivo positivo de los cambios electrocardiográficos de la onda $\mathrm{T}$ para una estenosis significativa de la ADA proximal de 69,89 y $86 \%$ respectivamente ${ }^{3}$.

Las anormalidades de la onda $\mathrm{T}$ son persistentes y pueden permanecer por horas a semanas, pudiendo ser encontradas cuando el paciente se encuentra libre de dolor. Con el manejo definitivo de la estenosis (angioplastía), los cambios electrocardiográficos evolucionan hacia la normalización del ECG.

Es fundamental reconocer estos cambios, asociándolos con una potencial obstrucción crítica de la ADA proximal y con un riesgo significativo para un IAM de pared anterior. 
Si bien la mayoría de los protocolos y guías clínicas para el manejo del IAM establecen elevación del segmento ST como piedra angular para la indicación de estrategias de reperfusión urgentes, es preciso una adecuada identificación de ciertos patrones electrocardiográficos que se han relacionado con oclusión aguda o estenosis crítica de una arteria coronaria ${ }^{4}$.

\section{Referencias}

1.- DE ZWAAN C, BÄR FW, WELLENS HJ. Characteristic electrocardiographic pattern indicating a critical stenosis high in the left anterior descending coronary artery in patients admitted because of impending myocardial infarction. Am Heart J 1982; 103: 730-6.

2.- RHINEHARDT J, BRADY WJ, PERRON AD, MATTU A. Electrocardiographic manifestations of Wellens' syndrome.
Am J Emerg Med 2002; 20: 638-43.

3.- HAINES DE, RAABE DS, GUNDEL WD, WACKERS FJ. Anatomic and prognostic significance of new T-wave inversion in unstable angina. Am J Cardiol. 1983; 52: 14-8.

4.- BIRNBAUM I, BIRNBAUM Y. High-risk ECG patterns in ACSNeed for guideline revision. J Electrocardiol. 2013; 46: 535-9. 